\title{
Applications of fuzzy inference systems in mineral industry-an overview
}

\author{
Michael Galetakis*, Anthoula Vasiliou, Emmanuel Steiakakis, Athanasia Soultana, and \\ Vassilios Deligiorgis \\ Technical University of Crete, School of Mineral Resources Engineering, University Campus, \\ Chania, Greece
}

\begin{abstract}
Recent advances in information and artificial intelligence technologies, and more specifically in Fuzzy Logic and Fuzzy Inference Systems (FIS), have provided a new approach in solving many problems related to mineral industry. The aim of the current study is to examine the application of FIS in mineral resources extractive industry by performing a recent literature review (2010-2020) of related studies published in engineering and earth science oriented scientific journals. Firstly the principles of Fuzzy Logic and the operation of FIS are briefly discussed and a descriptive example of a FIS used in mining with bucket wheel excavators is given. Secondly the results from the literature review are presented and the advantages as well as the trends in future development are discussed.
\end{abstract}

\section{Introduction}

Mineral industry operations are complicated and require an interdisciplinary approach presenting unique challenges in their design and implementation. Recent advances in Fuzzy Inference Systems (FIS) have provided a new approach in solving many problems related to mineral industry, a traditional economic activity which is heavily based on the experiential knowledge. The success of FIS is mainly due to their similarity to human perception and reasoning, and their intuitive handling and simplicity, which are important factors for the acceptance and usability of the mining systems. FIS have the ability to handle imprecise or incomplete information and to incorporate them into decision-making processes, based on the knowledge of an expert.

This paper examines the recent applications of FIS in the mineral industry, the obtained benefits, the emerged difficulties and the future trends. It is the successor (update and revised) of the first paper presented by Galetakis and Vasiliou in 2010 [3]. Initially the principles of fuzzy logic and fuzzy inference systems are given in section 2 and the development and operation of a FIS is presented through an illustrative example. Section 3 comprises a literature review of FIS application in the mineral industry. This literature review was based on engineering and earth science oriented journals available to Scopus scientific searching platform. The literature was divided into three broader categories corresponding to the major sections of mineral industry (exploration, mining and processing). The list of representative papers described in each category is not exhaustive and consequently the papers cited in each category do not comprise a complete bibliography of the available resources. Section 4 gives the advantages of the application of FIS in the mineral industry, as well as, the trends that seem to have developed over the last ten years across the different areas of mineral industry where fuzzy logic has been applied.

\footnotetext{
*Corresponding author: galetaki@mred.tuc.gr
} 


\section{Fuzzy logic and fuzzy inference systems}

\subsection{Basic principles of fuzzy logic and fuzzy inference systems}

Fuzzy logic, initiated in 1965, by Zadeh, is a multi-valued logic that allows intermediate values to be defined between conventional evaluations, like true/false, yes/no, high/low, etc. [28]. Notions like "rather thick" or "very thin" can be formulated mathematically and processed by computers, in order to apply a more human- -like way of thinking. Unlike the classical Boolean set allowing only 0 or 1 value, the fuzzy set is a set with a smooth boundary allowing partial membership. The degree of membership $\left(\mu_{\mathrm{A}}\right)$ in the set $\mathrm{A}$ is expressed by a number between 0 and 1 , with 0 indicating entirely not in the set $\mathrm{A}, 1$ indicating completely in the set $\mathrm{A}$ and a number in between meaning partially in the set $\mathrm{A}$. Figure 1 shows schematically the classification of a soil grain with diameter $D=1 / 17 \mathrm{~mm}$ when the conventional Boolean logic and the fuzzy logic is used. According to Boolean logic this grain is characterized as silt $\left(\mu_{\text {silt }}=1.0\right)$ while according to fuzzy logic this grain is characterized as silt with $\mu_{\text {silt }}=0.75$ and sand with $\mu_{\text {sand }}=0.25$

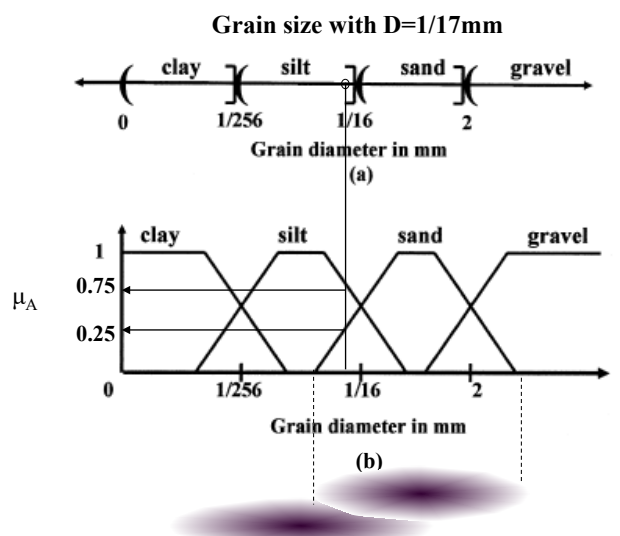

Fig. 1. Comparison of Boolean-set (a) versus fuzzy-set (b) representing the variable "grain size" (Modified from: Demico \& Klir, 2004).

FIS are computing frameworks, based on the concepts of fuzzy set theory and can be considered as a process for mapping a given input data set to an output set. The mineral industry sector has been particularly receptive to these methods, since many of the mining operations and processes are understood and controlled in empirical ways. The goal of the design of the fuzzy inference system is to capture the knowledge of a human expert relative to some specific domain and code this in a computer in such a way that the knowledge of the expert is available to a less experienced user [22].

The basic structure of a general FIS, shown in Figure 2, consists of three subsystems: a fuzzifier, a rule base and a defuzzifier. While the fuzzifier and the defuzzifier have the role of converting external information (crisp numbers) in fuzzy quantities and vice versa, the core of a FIS is its knowledge base, which is expressed in terms of fuzzy rules and allows for approximate reasoning $[2,3]$.

Fuzzy_Inference System

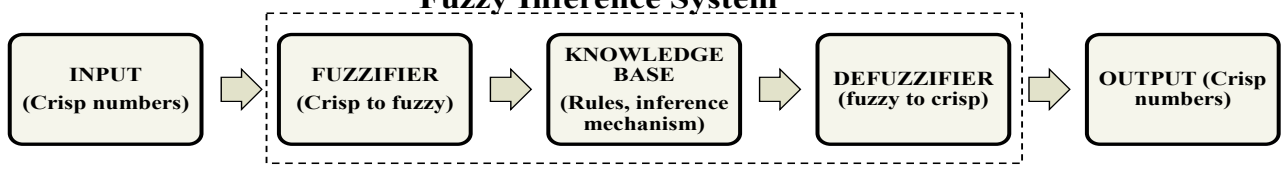

Fig. 2. Scheme of a typical Fuzzy Inference System. 
Fuzzification refers to the process of taking a crisp input value and transforming it into the degree required by the terms. The fuzzification subsystem measures the values of input variables, performs a scale mapping that transfers the range of values of input variables into corresponding universes of discourses and finally performs the function of fuzzification that converts input data into suitable linguistic values, which may be viewed as labels of fuzzy sets. Such a function is called the membership function and it is determined by the experts.

The knowledge base is consisting of a set of fuzzy "if-then" rules which capture the relation between input and output linguistic variables. From these rules and any fact describing actual states of input variables, the actual states of output variables are derived by an appropriate compositional rule of inference.

Typically, a FIS can be classified according to two main types of models that are distinguished in the formalization of the fuzzy rules: the Mamdani and the Takagi-SugenoKang models [1]. Mamdani FIS type was proposed as the first attempt to solve control problems, by a set of linguistic rules obtained from experienced human operators. The main feature of such type of FIS is that both the antecedents and the consequents of the rules are expressed as linguistic constraints. As a consequence, a Mamdani FIS can provide a highly intuitive knowledge base that is easy to understand and maintain, though its rule formalization requires a time consuming defuzzification procedure. Mamdani's FIS incorporates the following fuzzy rule schema:

IF $x$ is $A$ then $y$ is $B$ where, A and B are fuzzy sets defined on the input and output domains, respectively.

The Takagi-Sugeno-Kang (TSK) method of fuzzy inference generates the fuzzy rules from a given input-output data set in contrast to Madmani method which uses the knowledge of the expert to build the fuzzy set rules. The first two parts of the fuzzy inference process, fuzzifying the inputs and applying the fuzzy operator, are exactly the same in TSK and Mamdani FIS. The main difference between Mamdani and TSK is that the TSK output membership functions are either linear (first-order) or constant (zero-order). A typical rule for a two input-one output first order TSK fuzzy model has the form:

IF Input $1=\mathrm{x}$ and Input $2=\mathrm{y}$, THEN Output is $\mathrm{ax}+\mathrm{by}+\mathrm{c}$

For a zero-order Sugeno model, the output is a constant c since $\mathrm{a}=\mathrm{b}=0$.

FIS have been successfully applied in several engineering and scientific fields such as automatic control, data classification, decision analysis, expert systems and many others. According to Hoogendoorn et al., 1999, Sayers et al., 1995 and Sayers et al., 1996, the main advantages of a fuzzy logic approach are:

- Fuzzy systems can fuse quantitative and qualitative information and can exchange potentially conflicting objectives with the help of expert knowledge.

- Fuzzy systems are transparent, flexible and adaptable. The rule base and input variables used in fuzzy systems can be easily modified.

- Fuzzy systems are suitable for modelling complicate nonlinear problems.

\subsection{Application of Mamdani inference rules to the operation of bucket wheel excavators working in mine faces with hard rock inclusions}

In this example is described a FIS that aids the operator of a Bucket Wheel Excavator (BWE) equipped with geophysical sensors to avoid collisions of the excavating buckets with the hard rock inclusions and other problematic in digging materials, which can cause severe damages to BWE [5]. After comparison, ranking and evaluation via field testing of several geophysical methods identified that, in the typical geologic environment of lignite mines employing BWEs, the Electromagnetic Method (EM) measuring the electrical resistivity of the subsurface materials are the most promising in detecting local features such boulders and hard layers $[4,14,24]$. Thus a continuously working geophysical EM 
sensor mounted on the BWE scans the slope few cuts ahead of the face and measures the electrical resistivity of geological formations. The received data form EM sensor, after preprocessing and corrections, sent an automated algorithm to estimate the probability of occurrence of a hard rock formation at the position of measurement, and sends this information to expert system module. The developed expert system (FIS) uses the probability of occurrence of a hard rock formation and the operational data of the BWE to estimate the risk for collision and the diggability of the excavated material.

More specifically a Mamdani type FIS created, within Matlab programming environment. The development of the FIS included the selection of its inputs (the probability of occurrence of a hard rock formation at a specific position $\mathrm{S}$, the distance of bucket wheel to $\mathrm{S}$, the slewing speed of bucket wheel and the apparent resistivity values of the excavated material), the fuzzyfication-membership functions, the inference rules and the defuzzyfication method. Two outputs selected for the FIS, the risk for collision of the bucket wheel with a hard rock formation and the diggability of the excavated formation. The training of the developed FIS was based on collected data regarding structural and operational characteristics of the used BWEs and the applied mining practices. This information used to modify the structure and the inference rules of the FIS and to maximize the exploitation of the existing factual and experiential knowledge.

For the development of the FIS, the Fuzzy Logic Toolbox of the Mathworks was implemented [11]. The structure of the FIS, consisting of four inputs, two outputs and 12 rules, is shown in Figure 3. The use of fuzzy logic have allowed to capture and incorporate the existing experiential knowledge about excavated material properties and the mining with BWE in a very efficient way in the developed fuzzy expert system used to estimate the risk of collision of bucket wheel with hard rock formations. The use of fuzzy logic results in the gradual detection of collision and this was very convenient for BWE operators. Furthermore the developed fuzzy expert system can be simply adjusted through membership functions to include new factual knowledge while the fuzzy set of rules can be easily extended to incorporate additional experiential knowledge. This will allows the application of the developed system in different mining environments.

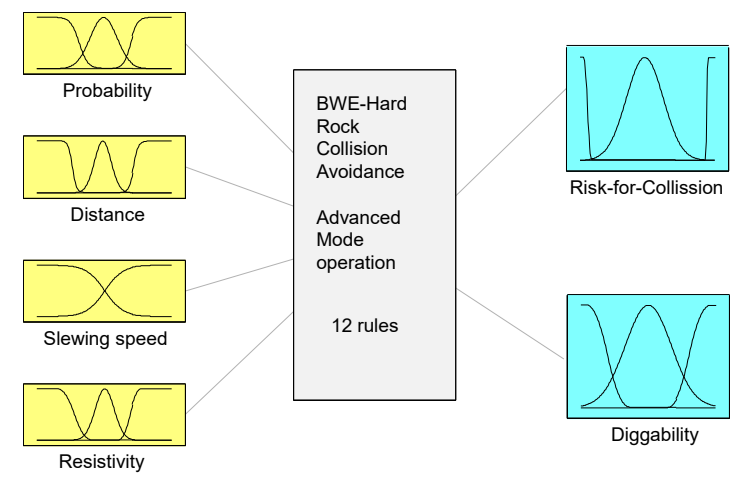

Fig. 3. Structure of development FIS in the Matlab programming environment (Fuzzy Logic Toolbox).

\section{Applications of fuzzy inference systems in mineral industry- a literature review}

In mineral industry sector, as already discussed, many processes are described and controlled in an empirical way. Because of this empirical culture of mining, fuzzy logic was 
able to proliferate quickly once a formal scientific basis was given by Zadeh in 1965 . Application of this fuzzy theory established a rationale for relative weights of importance used to evaluate deposits, to characterize rock masses, to select mining methods and excavation equipment, to design excavations, to control mineral processing operations and many others [12]. Thus it is not surprising that mineral processing engineering and other closely related areas have seen early and extensive use of fuzzy logic.

This review about the applications of fuzzy inference systems in the mineral industry was based on a literature survey covering the last decade, 2010-2020. Initially all engineering and earth and planetary sciences oriented journals, available to Scopus scientific searching platform, was used. The search terms included the words "fuzzy" and "mining industry". Then the results were refined and divided into three broader categories corresponding to the major sections of mineral industry (exploration, mining and processing). The analysis results are shown schematically in Figures 4 and 4. Figure 5 indicates the total number of the related articles as well as the articles published per year from 2010 to 2020. The rate of published articles shows clearly a significant increasing trend. The percentage of published articles in each category is given in Figure 5. The majority of FIS applications are related to mineral exploration and mining while those related to processing are fewer.

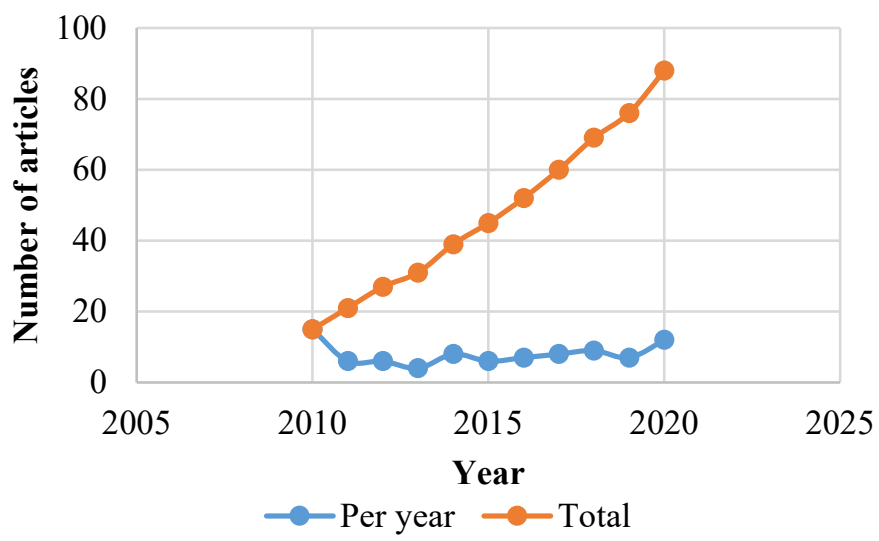

Fig. 4. Number of articles published in engineering and earth science oriented journal in the fields crossing the "fuzzy" and the "mining industry".

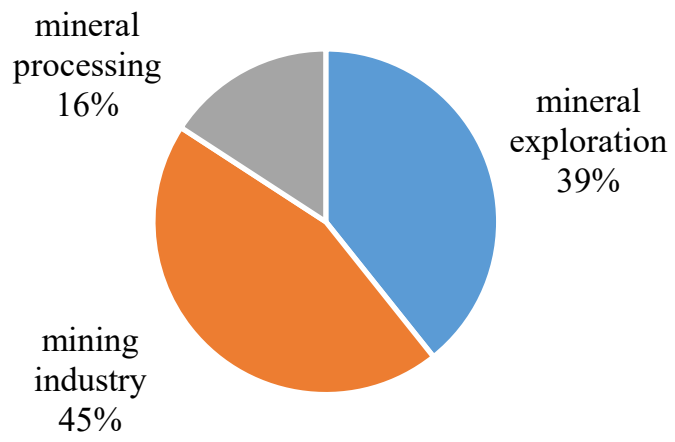

Fig. 5. Number of articles published in three categories representing the main mineral's industry activities. 
After this literature survey, representative articles from each category were selected and are presented below briefly. The list of representative papers described in each category is not exhaustive and consequently the papers presented in each category do not comprise a complete bibliography of the available resources.

\subsection{Applications of fuzzy inference systems in mineral exploration}

Guo et al., 2012, in their article: "Fuzzy comprehensive evaluation on relationship of coal maceral and porosity", investigated the relationships of coal maceral and porosity. By pressured-mercury testing and polarizing microscope analysis, it is found that vitrinite has higher content of transition pore and inertinite is rich of macroporous, meanwhile the specific pore volume of mesopore and microporous is related to both vitrinite and inertinite. It was found that the method of fuzzy comprehensive evaluation could be used to estimate the relationship of the coal maceral and porosity.

Parsa et al., 2017, in their article: "An improved data-driven fuzzy mineral prospectivity mapping procedure; cosine amplitude-based similarity approach to delineate exploration targets", a coherent set of exploration features recognized by using the distance distribution analysis. Then, the application of cosine amplitude-based similarity procedure adapted as a data-driven fuzzy logic approach for predictive mapping of porphyry-Cu prospectivity in Arasbaran metallogenic zone, NW Iran. In addition, a conventional datadriven fuzzy prospectivity model was generated for comparison purpose. Comparison of the two models demonstrated the superiority of the cosine amplitude-based fuzzy procedure for mineral prospectivity mapping.

Kim et al., 2019, in their article: "Application of fuzzy logic and geometric average: $\mathrm{A} \mathrm{Cu}$ sulfide deposits potential mapping case study from Kapsan Basin, DPR Korea", develop two kinds of knowledge-driven methods, one using the fuzzy logic and another using geometric average, applied to create the mineral potential maps for $\mathrm{Cu}$ sulfide deposits in the greenfield Kapsan Basin, DPR Korea. The ore geology studies for the study area have revealed that $\mathrm{Cu}$ sulfide deposits of hydrothermal genesis in Kapsan Basin are closely associated with Jurassic intrusions and faulting tectonics. Based on the conceptual model of $\mathrm{Cu}$ sulfide deposits and the available spatial datasets in the study area, used five independent evidential maps for $\mathrm{Cu}$ sulfide deposits potential mapping. The evidential map values were transformed into continuous values of the $[0,1]$ range using the non-linear fuzzy membership functions: logistic sigmoid and fuzzy Gaussian functions. Because the fuzzy logic and geometric average methods can use the same fuzzification methodology based on suitable membership functions, it is very economic and efficient to simultaneously apply two predictive models for mineral potential mapping of the study area. The preparation of these evidential layers were performed using spatial analyses supported in ArcGIS 10.4 GIS platform based on geological, geophysical and geochemical spatial datasets. The validation and comparative analysis results for the two predictive models demonstrated that most of known mineral occurrences are distributed in areas with high potential values. Both predictive models and their resulting potential maps are useful for evaluating the prospectivity of $\mathrm{Cu}$ sulfide deposits in Kapsan Basin.

Pahlavani et al., 2020, in their article: "Ranking potentially favorable mineralization zones using fuzzy VIKOR vs. Dempster-Shafer-fuzzy AHP methods, a case study: southeast of the Sarcheshmeh copper mine, Kerman, Iran", proposed a different ranking method to prioritize the mineral potential areas and select the best potential area to decrease potential risks of mineral exploration. At first, the area located in the east-southeast of the Sarcheshmeh copper mine was selected as mineral targets due to their high potential of porphyry copper occurrences. Then, the favorable porphyry copper areas were determined through collecting and integrating multiple exploratory evidential layers derived from remotely sensed imagery, geochemical, geophysical, lithological, and structural maps and 
images using the fuzzy logic approach in the GIS environment. Next, through building the decision matrix, the fuzzy VIKOR and Dempster-Shafer-fuzzy AHP methods were applied to estimate the favorability for copper porphyry deposits from information data layers, and the selected prospects were ranked and prioritized based on their scores obtained by each method. A comparison of the results obtained from each method with the previously discovered porphyry copper deposits and indications in the study area revealed a great match between the predicted and known deposits. The validation of results proved the ability of the proposed approach in detecting the highly favorable areas, particularly in the areas embedding known porphyry copper mineralizations.

\subsection{Applications of fuzzy inference systems in mining}

Yan et al., 2012, in their article: "Study on Early warning model of Coal mining engineering with Fuzzy AHP", analyzed the risk factors of coal mining engineering and studied the safety problem of "human-machine-environment" system. The risk factors of coal mining engineering divided to natural geological factors, personnel factors, equipment factors and management factors. Based on the fuzzy mathematics and these factors, the safety early warning model of coal mining engineering constructed by using the fuzzy AHP method. The results show that this early warning model is effective to prevent the accident.

Tripathy et al., 2018, in their article: "Risk Assessment in Underground Coalmines Using Fuzzy Logic in the Presence of Uncertainty", presented a method to assess safety risks in underground coalmines. The assessment of safety risks is based on the fuzzy reasoning approach. Mamdani fuzzy logic model is developed in the fuzzy logic toolbox of MATLAB. A case study is used to demonstrate the applicability of the developed model. The summary of risk evaluation in case study mine indicated that mine fire has the highest risk level among all the hazard factors. This study could help the mine management to prepare safety measures based on the risk rankings obtained.

Mottahedi and Ataei, 2019, in their article: "Fuzzy fault tree analysis for coal burst occurrence probability in underground coal mining", coal burst classified based its mechanisms into self-initiated and remotely triggered. Due to the sever coal burst consequences, its occurrence probability analysis is very essential. Combination of fuzzy theory and fault tree analysis used for coal burst occurrence probability analysis. Therefore, occurrence of coal burst as a top event in fault tree has been analyzed using fuzzy fault tree analysis. The critical paths that lead to coal burst are indicated in this article. These paths composed of important causing factors that known as basic events in fault tree. Therefore, high mining depth along with local mine stiffness lower than coal stiffness make a more critical path for self-initiated coal burst; and the seismic energy release due to the slippage of discontinuities nearby the coal mines make also a more critical path for remotely triggered coal burst.

Jiskani et al., 2020, in their article: "Assessment of risks impeding sustainable mining in Pakistan using fuzzy synthetic evaluation", assessed the risks that impede sustainable mining in Pakistan. A total of 41 risks were identified and categorized into eight categories based on expert consultation and literature review approaches. A risk assessment model based on the fuzzy synthetic evaluation was developed, which integrated data from 139 experienced mining professionals collected through surveys. The model calculated the probability of occurrence (P), and degree of impact (I) values to quantify scores for each risk factor, each risk category, and the overall risk level. To provide a basic idea for decision-makers to determine the most problematic risk categories that need to be managed on a case-by-case basis, these categories were classified into three priority levels according to their P and I values. The overall level of risk to sustainable mining was high, suggesting that the strategic focus should be on risk management. This study expands knowledge and literature on risks to sustainable mining that can help develop proper response strategies. 
Rui et al., 2020, in their article:" Critical factors to green mining construction in China: A two-step fuzzy DEMATEL analysis of state-owned coal mining enterprises", conducted an overall investigation on executed green mining policies and proposes an evaluation system of green mining construction. For the sake of clarity, all the related policies has been divided into 5 categories including financial factors, regulatory factors, organizational factors, social factors and technical factors. To disentangle the interrelations between factors and ascertain the critical factors, an improved two-step fuzzy DEMATEL model is adopted for assessment with group knowledge from several long-term and experienced experts in state-owned coal mining enterprises. Results reveal that the regulatory factors and organizational factors, especially the political pressure of local governments and the administrative ranking of enterprise leader, are the critical factors to green mining construction in China, and the social factors are relatively insignificant.

\subsection{Applications of fuzzy inference systems in mineral processing}

$\mathrm{Wu}$ and Chai, 2010, in their article: "Soft sensing method for magnetic tube recovery ratio via fuzzy systems and neural networks", proposed a new soft sensing method, which uses fuzzy system and neural network techniques. The contributions of their soft sensing method are that a fuzzy mechanism model for magnetic tube recovery ratio (MTRR) is used, which is obtained from data clustering. They do not update the fuzzy model, but use a neural compensator to improve the modeling accuracy, where the training algorithm for the neural network stable. This soft sensing method has been successfully applied in a metal company in China.

Piltan et al., 2012, in their article: "Proposing a decision-making model using analytical hierarchy process and fuzzy expert system for prioritizing industries in installation of combined heat and power systems", proposed a hybrid decision making model for prioritizing Iranian industries to install combined heat and power (CHP) systems. Integrated analytical hierarchy process (AHP) and fuzzy expert system (FES) applied. Weighted amount of each decision criterion which calculated by AHP affects the fuzzy membership function in FES model. In this model, 23 Iranian industries (based on international standard industrial classification) ranked using the combination of AHP and FES considering weighted criteria and actual data. The results demonstrate that the priority of chemical, non-metallic minerals, refined petroleum, food products and beverages, basic metals, paper and paper products, and textile industries, for installation of CHP is more than other industries.

Van Zyl et al., 2013, in their article: "Implementation of a SAG Grinding Expert System at Kansanshi Mine - Zambia", described the method and results of the advanced control system that implemented on the Sulphide milling circuit. The acceptance and performance achieved may be attributed to the approach in implementing the system, as well as the configuration of the fuzzy logic control strategy. First Quantum's Kansanshi Mine and the Advanced Systems Group of SGS collaborated on the implementation of an expert system for the control and optimisation of a Semi-Autogenous (SAG) mill on the sulphide grinding circuit. The primary objective was to increase SAG mill stability and to maximise throughput. This was achieved by optimisation of the mill load and power draw by the manipulation of the feed, speed and density in the mill. The SGS expert control technology has proven its success at achieving these objectives in a variety of different processes that were prone to a high degree of variability.

Hadizadeh et al., 2017, in their article: "Supervisory Fuzzy Expert Controller for Sag Mill Grinding Circuits: Sungun Copper Concentrator", presented the basis of a supervisory fuzzy expert controller for Semi-Autogenous Grinding (SAG) mill circuits. Although leading companies in control and automation have their own commercial packages, this supervisory controller coded in Matlab using Mamdani method and is able to connect to 
plant lower level control system. In the proposed controller, fuzzy system calculates optimum set points to the plant Distributed Control Systems (DCS) control loops, enabling them to change the manipulating parameters to reach the new set points. This controller installed, tested and verified in a copper grinding circuit. Results showed $1.8 \%$ increase in mill throughput, $3 \%$ decrease in power draw and more stable feeding regime at the same time.

Sitorus and Brito-Parada, 2020, in their article: "Equipment selection in mineral processing - A sensitivity analysis approach for a fuzzy multiple criteria decision making model", proposed a new sensitivity analysis approach by applying an additional fuzzification factor and disagreement level of decision makers in order to model uncertainty. For this purpose, a case study for the selection of primary crushers was considered. Five types of primary crushers were evaluated with respect to six criteria to showcase the applicability of the proposed approach to assess integrated constrained fuzzy stochastic analytic hierarchy process (IC-FSAHP). The results obtained showcase that the proposed sensitivity analysis approach is capable of providing extensive and useful "whatif" information on the decision making results.

\section{Conclusions - Future developments}

From the literature review it is clear to be a major trend in the use of fuzzy logic in the fields of mineral exploration, mining and mineral processing. Initially, fuzzy-based systems were used to capture the experiential knowledge and to handle the geological uncertainty. FIS superiority over conventional systems is based on their capability to acquire knowledge from mining engineers, geologists, operators, and other experts and to represent and manipulate it in expert systems in a human-like manner.

More specifically, in the area of mineral exploration the main advantages of FIS are their ability to capture easily and effectively the geologic knowledge and to describe geological variables which are usually expressed in natural language. FIS combined with Geographic Information Systems can be used as a framework to store, manage and analyze crisp and uncertain data obtained during the mineral exploration, such as data from remote sensing, from geophysical, geochemical, geological surveys and drillhole data. In mining and mineral processing FIS can be used to select the most suitable mining method, to improve the efficiency of mining equipment during excavation and to control effectively mineral processing plants. The transparency, intuitive nature, flexibility, and short response time of FIS, make them unique for controlling processes such crushing or froth flotation. Moreover fuzzy logic-based controllers are suitable for on-line monitoring systems (assaying from mineral streams) and for fault detection.

Many fuzzy-based systems were also used in combination with previous developed deterministic models. These hybrid fuzzy systems are combinations of knowledge-based and model-based fuzzy systems. At this time knowledge-based fuzzy systems are more developed than model-based or hybrid fuzzy systems. Another interesting approach that is growing rapidly with remarkable potential for applications in mineral industry is the fusion of fuzzy systems, artificial neural networks and genetic algorithms.

\section{References}

1. G. Castellano, A.M. Fanelli, C. Mencar, Generation of interpretable fuzzy granules by a double-clustering technique. Archives of Control Sciences: Special issue on Granular Computing 12 (4), 397-410 (2002) 
2. E. Czogala, J. Leski, Fuzzy and neuro-fuzzy intelligent systems, Physica-Verlag (2000)

3. M. Galetakis, A. Vasiliou, Applications of fuzzy inference systems in mineral industry- overview. Book chapter in Expert System Software, editors: J. M. Segura and A. C. Reiter, 211-217 (2010)

4. M. Galetakis, T. Michalakopoulos, A. Bajcar, C. Roumpos, M. Lazar, P. Svoboda, Project BEWEXMIN: Bucket wheel excavators operating Under difficult mining conditions including unmineable Inclusions and geological structures with excessive mining resistance, Proceedings of 13th International Symposium Continuous Surface Mining, ISCSM 2016, 103-114 (2016)

5. M. Galetakis, A. Vafidis, A. Vasiliou, G. Kritikakis, V. Deligiorgis, T. Michalakopoulos, G. Apostolopoulos, C. Roumpos, F. Pavloudakis, Development of a fuzzy inference system for avoiding collision of bucket wheel excavator equipped with electromagnetic (EM) sensors with hard rock inclusions, Górnictwo Odkrywkowe, 59 4, 16-22 (2018)

6. L. Guo, Y. Qi, Y. Wang, F. Yang, Fuzzy comprehensive evaluation on relationship of coal maceral and porosity, International Symposium on Safety Science and Technology, Procedia Engineering 45, 962-966 (2012)

7. M. Hadizadeh, A. Farganegan, M. Noaparast, Supervisory Fuzzy Expert Controller for Sag Mill Grinding Circuits: Sungun Copper Concentrator, Mineral Processing and Extractive Metallurgy Review, 38:3, 168-179 (2017)

8. S. Hoogendoorn, S. Hoogendoorn-Lanser, H. Schuurman, Fuzzy perspectives in traffic engineering, Workshop on Intelligent Traffic Management Models, (1999)

9. I.M. Jiskani, Q. Cai, W. Zhou, X. Lu, Assessment of risks impeding sustainable mining in Pakistan using fuzzy synthetic evaluation, Resources Policy 69, 1-13 (2020)

10. Y. Kim, K. Choe, R. Ri, Application of fuzzy logic and geometric average: $\mathrm{A} \mathrm{Cu}$ sulfide deposits potential mapping case study from Kapsan Basin, DPR Korea, Ore Geology Reviews 107, 239-247 (2019)

11. Mathworks Inc, Fuzzy Logic Toolbox for use with Matlab, User's Guide version 2 (1999)

12. J. Meech, The evolution of intelligent systems in the mining industry, In Proceedings of the international conference on mineral process modeling, simulation and control, 1-30 (2006)

13. A. Mottahedi, M. Ataei, Fuzzy fault tree analysis for coal burst occurrence probability in underground coal mining. Tunnelling and Underground Space Technology 83, 165-174 (2019)

14. L. Overmeyer, M. Kesting, K. Jansen, SIMT Technology - Sensory identification of material type and detection of the interfaces, Bulk Solids Handling, 27 (2), 112118 (2007)

15. P. Pahlavani, S. Riahi, B. Bidgeli, Ranking potentially favorable mineralization zones using fuzzy VIKOR vs. Dempster-Shafer-fuzzy AHP methods, a case study: southeast of the Sarcheshmeh copper mine, Kerman, Iran. Arabian journal of Geosciences 13:1167, 1-21 (2020)

16. M. Parsa, A. Maghsoudi, M. Yousefi, An improved data-driven fuzzy mineral prospectivity mapping procedure; cosine amplitude-based similarity approach to delineate exploration targets, International Journal of Applied Earth Observation and Geoinformation 58, 157-167 (2017) 
17. M. Piltan, E. Mehmanchi, S.F. Ghaderi, Proposing a decision-making model using analytical hierarchy process and fuzzy expert system for prioritizing industries in installation of combined heat and power systems, Expert Systems with Applications 39, 1124-1133 (2012)

18. R. Qi, S. Li, L. Qu, L. Sun, C. Gong, Critical factors to green mining construction in China: A two-step fuzzy DEMATEL analysis of state-owned coal mining enterprises, Journal of Cleaner Production 273, 1-14 (2020)

19. T.M. Sayers, M.G.H. Bell, T. Mieden, F. Busch, Improving the traffic responsiveness of signal controllers using fuzzy logic, IEE Colloquium on Urban Congestion Management 207, 6/1-6/4 (1995)

20. T.M. Sayers, M.G.H. Bell, T. Mieden, F. Busch, Traffic responsive signal control using fuzzy logic - a practical modular approach, Proceedings of the 1996 IEE Colloquium on Fuzzy Logic Controllers In Practice 200, 5/1-5/4 (1996)

21. F. Sitorus, P.R. Brito-Parada, Equipment selection in mineral processing - A sensitivity analysis approach for a fuzzy multiple criteria decision making model, Minerals Engineering 150, 1-8 (2020)

22. K.A. Tripathi, Review on Knowledge-based Expert System: Concept and Architecture, IJCA Special Issue on "Artificial Intelligence Techniques - Novel Approaches \& Practical Applications", 19-23 (2011)

23. D. P. Tripathi, C.K. Ala, Risk Assessment in Underground Coalmines Using Fuzzy Logic in the Presence of Uncertainty, Journal of The Institution of Engineers (India): Series D, 99, 157-163 (2018)

24. A. Vafidis, N. Economou, M. Galetakis, A. Vasiliou, T. Michalakopoulos, G. Apostolopoulos, Assessing the potential of ground penetrating radar (GPR) to detect hard geological formations and inclusions during the excavation by bucket-wheel excavators, Proceedings of 13th International Symposium Continuous Surface Mining, ISCSM 2016, 631-643 (2016)

25. F. Van Zyl, F. Paquot, F. Metzner, Fouche, A. Gomez, Implementation of a SAG Grinding Expert System at Kansanshi Mine - Zambia, 16th IFAC Symposium on Automation in Mining, Mineral and Metal Processing 46, Issue 16, 176-181 (2013)

26. F. Wu, T. Chai, Soft sensing method for magnetic tube recovery ratio via fuzzy systems and neural networks. Neurocomputing 73, 2489-2497 (2010)

27. Z. Yan, X. Wang, Y. Fu, Study on Early warning model of Coal mining engineering with Fuzzy AHP, Systems Engineering Procedia 5, 113-118 (2012)

28. L.A. Zadeh, Toward a theory of fuzzy information granulation and its centrality in human reasoning and fuzzy logic, Fuzzy Sets and Systems 90, 111-117 (1997) 